\title{
LYAPUNOV FUNCTIONS FOR LINEAR NONAUTONOMOUS DYNAMICAL EQUATIONS ON TIME SCALES
}

\author{
PETER E. KLOEDEN AND ALEXANDRA ZMORZYNSKA
}

Received 25 January 2006; Revised 23 March 2006; Accepted 13 April 2006

The existence of a Lyapunov function is established following a method of Yoshizawa for the uniform exponential asymptotic stability of the zero solution of a nonautonomous linear dynamical equation on a time scale with uniformly bounded graininess.

Copyright (c) 2006 P. E. Kloeden and A. Zmorzynska. This is an open access article distributed under the Creative Commons Attribution License, which permits unrestricted use, distribution, and reproduction in any medium, provided the original work is properly cited.

\section{Introduction}

Lyapunov functions are a very useful tool for investigating the behaviour of dynamical equations. They have been used now for over a century for differential equations of many types [15] as well as difference equations [1]. They were first used in the context of time scales in [12]. See [16] for a more recent and an extensive investigation of Lyapunov functions on time scales.

Much of the literature on Lyapunov functions, especially applications oriented, deals with sufficient conditions, assuming that a Lyapunov function is known. An important theoretical issue with practical implications is whether or not a Lyapunov function characterizing a particular dynamical property actually exists-such results are known as necessary conditions.

In this paper, we establish the existence of a Lyapunov function characterising the uniform exponential asymptotic stability of the zero solution of a nonautonomous linear dynamic equation

$$
x^{\Delta}=A(t) x
$$

on a time scale $\mathbb{T}$ with a bounded graininess, where the matrix-valued mapping $t \mapsto A(t)$ is right dense continuous (rd-continuous) on $\mathbb{T}$, that is, $A \in \mathscr{C} \operatorname{rd} \mathscr{R}\left(\mathbb{T}, \mathbb{R}^{n \times n}\right)$.

Such linear dynamical equations and their inhomogeneous variants play an important role in investigations of the dynamical behaviour, both in themselves and also as 
linearizations of nonlinear systems, see, for example, $[3,7,11]$. We note that Pötzsche et al. [13] investigated various necessary and sufficient conditions for the uniform exponential stability of the zero solution of systems (1.1) in terms of spectral properties rather than Lyapunov functions.

In the next section, we show the invertibility of the cylinder transformation on the Hilger complex plane. This is required in our main result, which is presented and proved in the final section.

Background concepts and results on time scales are taken from Bohner and Peterson [4] (see also $[2,5,8,9]$ ) and, for brevity, will not be stated explicitly here in general.

\section{Invertibility of the cylinder transformation}

A classical result from function theory (see, e.g., [6, Pages 38-45]) says that the main branch of the complex $\operatorname{logarithm} \log (z):=\log (|z|)+\imath \phi$, where $z=|z| e^{\imath \phi}$ with $-\pi<\phi \leq$ $\pi$, is well defined for $z \in \mathbb{C}^{*}:=\mathbb{C} \backslash\{0\}$, but is not continuous for $z \in \mathbb{C}^{*}$ with $\operatorname{Im}(z)=\pi$. Moreover, $\log (z)$ is a holomorphic function for $z \in \mathbb{C}^{-}:=\mathbb{C} \backslash\{z \in \mathbb{R}: z \leq 0\}$.

Define $\mathbb{C}_{h}$ and $\mathbb{Z}_{h}$ for $h>0$ by

$$
\mathbb{C}_{h}:=\left\{z \in \mathbb{C}: z \neq-\frac{1}{h}\right\}, \quad \mathbb{Z}_{h}:=\left\{z \in \mathbb{C}:-\frac{\pi}{h}<\operatorname{Im}(z)<\frac{\pi}{h}\right\} .
$$

$\mathbb{C}_{h}$ is called the Hilger complex plane. Hilger [10] also introduced the cylinder transformation $\xi_{h}: \mathbb{C}_{h} \rightarrow \mathbb{Z}_{h}$, which is defined by

$$
\xi_{h}(z):= \begin{cases}\frac{1}{h} \log (1+z h), & h>0 \\ z, & h=0 .\end{cases}
$$

Proposition 2.1 (Hilger [10]). Let $\mathbb{T}$ be a time scale with graininess $\mu$ and let $p \in \mathscr{C}^{r d}(\mathbb{T}, \mathbb{C})$ be regressive on $\mathbb{T}$. Then the composition $\xi_{\mu}(p)$ of the cylinder transformation with $\mu$ and $p$ is well defined, $r d$-continuous on $\mathbb{T}$, and thus Cauchy integrable.

The cylinder transformation is useful for investigating, among other things, linear dynamical equations. It is invertible when suitably restricted.

Lemma 2.2. Let $\mathbb{A}_{h}:=\left\{z \in \mathbb{C}_{h}: z<-1 / h\right\}$ for $h>0$ and define $\xi_{h}: \mathbb{C}_{h} \backslash \mathbb{A}_{h} \rightarrow \mathbb{Z}_{h}$ by

$$
\xi_{h}(z):=\frac{1}{h} \log (1+z h)
$$

Then $\xi_{h}$ is a biholomorphism of $\mathbb{C}_{h} \backslash \mathbb{A}_{h}$ onto $\mathbb{Z}_{h}$ and its inverse $\xi_{h}^{-1}: \mathbb{Z}_{h} \rightarrow \mathbb{C}_{h} \backslash \mathbb{A}_{h}$ is given by

$$
\xi_{h}^{-1}(z):=\frac{\exp (z h)-1}{h}
$$

Proof. Since $\xi_{h}$ and $\xi_{h}^{-1}$ are well defined and continuous, it remains to prove that

(1) $\xi_{h}\left(\mathbb{C}_{h} \backslash \mathbb{A}_{h}\right) \subset \mathbb{Z}_{h}$ and $\xi_{h}^{-1}\left(\xi_{h}(z)\right)=z$ for all $z \in \mathbb{C}_{h} \backslash \mathbb{A}_{h}$. 
Let $z \in \mathbb{C}_{h} \backslash \mathbb{A}_{h}$. Then $1+z h \in \mathbb{C}^{-}$and

$$
\xi_{h}(z)=\frac{1}{h} \log (1+z h)=\frac{\log |1+z h|}{h}+i \cdot \frac{\operatorname{Arg}(1+z h)}{h}
$$

with $-\pi<\operatorname{Arg}(1+z h)<\pi$, from which it follows that

$$
-\frac{\pi}{h}<\frac{\operatorname{Arg}(1+z h)}{h}<\frac{\pi}{h}
$$

hence $\xi_{h}\left(\mathbb{C}_{h} \backslash \mathbb{A}_{h}\right) \subset \mathbb{Z}_{h}$. Furthermore, a classical result from function theory says that $\exp (\log (1+z h))=1+z h$ for $z \in \mathbb{C}_{h} \backslash \mathbb{A}_{h}$ and $h>0$ (see, e.g., [14, Pages 120 and 126]). Hence

$$
\xi_{h}^{-1}\left(\xi_{h}(z)\right)=\frac{\exp (h \cdot(1 / h) \log (1+z h))-1}{h}=\frac{1+z h-1}{h}=z .
$$

(2) $\xi_{h}^{-1}\left(\mathbb{Z}_{h}\right) \subset \mathbb{C}_{h} \backslash \mathbb{A}_{h}$ and $\xi_{h}\left(\xi_{h}^{-1}(z)\right)=z$ for all $z \in \mathbb{Z}_{h}$.

We assume that there exists a $z \in \mathbb{Z}_{h}$ such that for $h>0$

$$
\exp (z h) \leq-\frac{1}{h}
$$

Applying Euler's formula we see that (2.8) holds for $z \in \mathbb{Z}_{h}$ if and only if $\operatorname{Im}(z)=0$, that is, $z \in \mathbb{R}$. But then a contradiction follows directly from (2.8), since the exponential function is strictly positive on $\mathbb{R}$. Hence $\xi_{h}^{-1}\left(\mathbb{Z}_{h}\right) \subset \mathbb{C}_{h} \backslash \mathbb{A}_{h}$. We have $-\pi / h<\operatorname{Im}(z)<\pi / h$ whenever $z \in \mathbb{Z}_{h}$. It then follows from [14, Pages 120 and 126] that $\log (\exp (z h))=z h$ and hence

$$
\frac{1}{h} \log \left(1+h \cdot \frac{\exp (z h)-1}{h}\right)=\frac{1}{h} \log (\exp (z h))=z
$$

for $z \in \mathbb{Z}_{h}$ and $h>0$.

We have shown that $\xi_{h}: \mathbb{C}_{h} \backslash \mathbb{A}_{h} \rightarrow \mathbb{Z}_{h}^{\prime}$ is bijective with inverse function $\xi_{h}^{-1}: \mathbb{Z}_{h} \backslash \mathbb{A}_{h}$. From function theory, it is known that exp is a holomorphism on $\mathbb{C}$ and that Log is a holomorphism on $\mathbb{C}^{-}$(see, e.g., [14, Page 64]). Hence $\xi_{h}$ is a holomorphism on $\mathbb{C}_{h} \backslash \mathbb{A}_{h}$ and $\xi_{h}^{-}$is a holomorphism on $\mathbb{Z}_{h}$ for $h>0$.

Remark 2.3. Write $\mathbb{C}_{0}:=\mathbb{C}$ for $h=0$. Then we define $\xi_{0}:=$ id on $\mathbb{C}$ and $\xi_{0}^{-1}:=$ id on all of $\mathbb{C}$, where id is the identity function on $\mathbb{C}$.

For fixed $c \in \mathbb{R}$, the composition of the inverse cylinder transformation with the graininess $\mu$ of a time scale $\mathbb{T}$ will be needed in considering Lyapunov functions. Let $c \in \mathbb{R}$ be fixed and $\bar{\xi}_{c}: \mathbb{T} \rightarrow \mathbb{R}$ be defined by

$$
\bar{\xi}_{c}(t):= \begin{cases}\frac{\exp (\mu(t) \cdot c)-1}{\mu(t)}, & t<\sigma(t) \\ c, & t=\sigma(t) .\end{cases}
$$


4 Lyapunov functions for dynamical equations

Then

(i) the function $t \mapsto \bar{\xi}_{c}(t)$ is rd-continuous in all $t \in \mathbb{T}$ for arbitrary $c \in \mathbb{R}$ as the composition of the graininess function $t \mapsto \mu(t)$, which is always rd-continuous (see [4, Example 1.59 and Theorem 1.60, Page 22]) and the continuity of $f: \mathbb{R} \rightarrow$ $\mathbb{R}$ defined by

$$
f(x):= \begin{cases}\frac{\exp (x)-1}{x}, & x \neq 0 \\ 1, & x=0\end{cases}
$$

(ii) since $f(x)>0$ for all $x \in \mathbb{R}$, for $c>0$, it follows that $\bar{\xi}_{c}(t)>0$ for all $t \in \mathbb{T}$ and for $c<0$, it follows that $\bar{\xi}_{c}(t)<0$ for all $t \in \mathbb{T}$;

(iii) moreover, $\bar{\xi}_{c}(t)=c$ for all right dense $t \in \mathbb{T}$;

(iv) for $h>0$, let $\mathbb{T}:=h \mathbb{Z}$, then $\mu(t)=h$ and

$$
\bar{\xi}_{c}(t)=\frac{\exp (h \cdot c)}{h}
$$

for all $t \in \mathbb{T}$, that is, $t \mapsto \bar{\xi}_{c}(t)$ is a constant function.

\section{Existence of a Lyapunov function}

Our main result, the following theorem, is a necessary condition for the existence of a Lyapunov function characterizing the uniform exponential asymptotic stability of the zero solution of a nonautonomous linear dynamical equation. It is adapted from Yoshizawa [15, Theorem 19.1] for linear ordinary differential equations.

Theorem 3.1. Let $\mathbb{T}$ be an unbounded time scale with bounded graininess and suppose that for each $t_{0} \in \mathbb{T}, x_{0} \in \mathbb{R}^{n}$, there exists a classical solution $y: \mathbb{T} \rightarrow \mathbb{R}^{n}, y(t)=y\left(t, t_{0}, x_{0}\right)$ of the initial value problem (IVP)

$$
\begin{gathered}
y^{\Delta}=A(t) y, \quad A \in \mathscr{C}^{r d \mathscr{R}}\left(\mathbb{T}, \mathbb{R}^{n \times n}\right), t \in \mathbb{T}, \\
y\left(t_{0}\right)=x_{0}, \quad x_{0} \in \mathbb{R}^{n}, t_{0} \in \mathbb{T},
\end{gathered}
$$

for all $t \in \mathbb{T}, t \geq t_{0}$. Furthermore, suppose that there exist constants $K>0$ and $c>0$, which can be chosen independently of $t_{0}$ such that

$$
\left\|y\left(t, t_{0}, x_{0}\right)\right\| \leq K e^{-c\left(t-t_{0}\right)}\left\|x_{0}\right\|
$$

holds for any $t_{0} \in \mathbb{T}, x_{0} \in \mathbb{R}^{n}$, and all $t \geq t_{0}$, that is, the zero solution is uniformly exponentially stable.

Then there exists a function $V: \mathbb{T} \times \mathbb{R}^{n} \rightarrow \mathbb{R}$ such that

(i) $\|x\| \leq V(t, x) \leq K\|x\|$ for all $x \in \mathbb{R}^{n}, t \in \mathbb{T}$;

(ii) $|V(t, x)-V(t, \tilde{x})| \leq K\|x-\tilde{x}\|$ for any fixed $t \in \mathbb{T}$ and all $x, \tilde{x} \in \mathbb{R}^{n}$; 
(iii) for arbitrary fixed $\left(t_{0}, x_{0}\right) \in \mathbb{T} \times \mathbb{R}^{n}$, define function $V_{*}: \mathbb{T} \rightarrow \mathbb{R}, V_{*}(t):=V(t, y(t))$, where $y(t)=y\left(t, t_{0}, x_{0}\right)$, then the upper right Dini derivative of $V_{*}$ defined by

$$
V_{*}^{\Delta}(t):= \begin{cases}\varlimsup_{h \backslash 0, h+t \in \mathbb{T}} \frac{V_{*}(t+h)-V_{*}(t)}{h}, & \text { if } t=\sigma(t), \\ \frac{V_{*}(\sigma(t))-V_{*}(t)}{\mu(t)}, & \text { if } t<\sigma(t),\end{cases}
$$

exists and the estimate

$$
V_{*}^{\Delta}(t) \leq \bar{\xi}_{-c}(t) V_{*}(t)<0
$$

holds for all $t \geq t_{0}$;

(iv) $V$ is continuous from the right in $(t, x) \in \mathbb{T} \times \mathbb{R}^{n}$, that is,

$$
\lim _{(\tilde{t}, \tilde{x}) \rightarrow(t, x), \tilde{t} \geq t}|V(\tilde{t}, \tilde{x})-V(t, x)|=0
$$

Proof. Fix $t \in \mathbb{T}$. Then we define

$$
A_{t}:=\{\tau \in[0, \infty): t+\tau \in \mathbb{T}\}
$$

since $0 \in A_{t}$, we note that $A_{t}$ is nonempty. Further, we define $V: \mathbb{T} \times \mathbb{R}^{n} \rightarrow \mathbb{R}$ by

$$
V(t, x):=\sup _{\tau \in A_{t}}\|y(t+\tau, t, x)\| e^{c \tau}
$$

where $y: \mathbb{T} \rightarrow \mathbb{R}^{n}$ is the unique solution of (3.1) with initial value $y(t)=x$. Equation (3.7) is well defined since $y$ exists for all initial values $(t, x) \in \mathbb{T} \times \mathbb{R}^{n}$ and all $t+\tau, \tau \in A_{t}$ by assumption and, as we will see in detail, it also follows from the assumptions that $V$ is bounded.

It will be shown that $V$ satisfies the properties (i)-(iv) asserted in the theorem.

It is obvious from the definition of $V$ that $\|x\| \leq V(t, x)$, while from (3.2) it follows for all $\tau \in A_{t}$ that

$$
\|y(t+\tau, t, x)\| \leq K e^{-c(t+\tau-t)}\|x\|=K e^{-c \tau}\|x\|,
$$

so

$$
V(t, x)=\sup _{\tau \in A_{t}}\|y(t+\tau, t, x)\| e^{c \tau} \leq \sup _{\tau \in A_{t}} K e^{-c \tau}\|x\| e^{c \tau}=K\|x\|
$$

Thus $V$ satisfies property (i). 
Let $t \in \mathbb{T}$ be fixed and let $x, \tilde{x} \in \mathbb{R}^{n}$ be arbitrary. By the triangle inequality and the superposition of solutions of a linear system, we have

$$
\begin{aligned}
|V(t, x)-V(t, \tilde{x})| & =\left|\sup _{\tau \in A_{t}}\left\|y(t+\tau, t, x)|| e^{c \tau}-\sup _{\tau \in A_{t}}\right\| y(t+\tau, t, \tilde{x}) \| e^{c \tau}\right| \\
& =\left|\sup _{\tau \in A_{t}} e^{c \tau}(\|y(t+\tau, t, x)\|-\|y(t+\tau, t, \tilde{x})\|)\right| \\
& \leq\left|\sup _{\tau \in A_{t}} e^{c \tau}(\|y(t+\tau, t, x)-y(t+\tau, t, \tilde{x})\|)\right| \\
& \leq \sup _{\tau \in A_{t}} e^{c \tau}|| y(t+\tau, t, x-\tilde{x})\|=V(t, x-\tilde{x}) \leq K\| x-\tilde{x} \|,
\end{aligned}
$$

which shows that $V$ is globally Lipschitz-continuous in $x$ for fixed $t \in \mathbb{T}$, that is, satisfies property (ii).

We will prove property (iii) next. Let $\left(t_{0}, x_{0}\right) \in \mathbb{T} \times \mathbb{R}^{n}$ be arbitrary. We will distinguish two cases, $\sigma(t)=t$ and $\sigma(t)>t$ in the proof.

Suppose that $\sigma(t)=t$ and let $h \in A_{t}$. Then

$$
V_{*}^{\Delta}(t)=\varlimsup_{h \backslash 0, h \in A_{t}} \frac{V_{*}(t+h)-V_{*}(t)}{h},
$$

where, by the uniqueness of solutions of (3.1) (see [4, Theorem 8.20, Page 324]), it follows that

$$
\begin{aligned}
V_{*}(t+h) & =\sup _{\tau \in A_{t+h}}\left\|y\left(t+h+\tau, t+h, y\left(t+h, t_{0}, x_{0}\right)\right)\right\| e^{c \tau} \\
& =\sup _{\tau \in A_{t+h}}\left\|y\left(t+h+\tau, t, y\left(t, t_{0}, x_{0}\right)\right)\right\| e^{c \tau} \\
& =\sup _{\tau \in\{\tau \in[h, \infty): t+\tau \in \mathbb{T}\}}\left\|y\left(t+\tau, t, y\left(t, t_{0}, x_{0}\right)\right)\right\| e^{c \tau} e^{-c h} \\
& \leq \sup _{\tau \in\{\tau \in[0, \infty): t+\tau \in \mathbb{T}\}}\left\|y\left(t+\tau, t, y\left(t, t_{0}, x_{0}\right)\right)\right\| e^{c \tau} e^{-c h} \\
& =V_{*}(t) e^{-c h},
\end{aligned}
$$

hence we have

$$
V_{*}^{\Delta}(t) \leq \varlimsup_{h \backslash 0} V_{*}(t) \cdot \frac{e^{-c h}-1}{h}=V_{*}(t) \cdot(-c)=V_{*}(t) \cdot \bar{\xi}_{-c}(t),
$$

since $\mu(t)=0$ by assumption.

Now suppose that $\sigma(t)>t$, in which case

$$
V_{*}^{\Delta}(t)=\frac{V_{*}(\sigma(t))-V_{*}(t)}{\mu(t)} .
$$


But

$$
\begin{aligned}
V_{*}(\sigma(t)) & =\sup _{\tau \in A_{\sigma(t)}}\left\|y\left(\sigma(t)+\tau, \sigma(t), y\left(\sigma(t), t_{0}, x_{0}\right)\right)\right\| e^{c \tau} \\
& =\sup _{\tau \in A_{\mu(t)+t}}\left\|y\left(\mu(t)+t+\tau, \mu(t)+t, y\left(\mu(t)+t, t_{0}, x_{0}\right)\right)\right\| e^{c \tau} \\
& =\sup _{\tau \in\{\tau \in[\mu(t), \infty): t+\tau \in \mathbb{T}\}}\left\|y\left(t+\tau, t, y\left(t, t_{0}, x_{0}\right)\right)\right\| e^{c \tau} e^{-\mu(t) c} \\
& \leq V_{*}(t) e^{-c \mu(t)},
\end{aligned}
$$

so

$$
V_{*}^{\Delta}(t) \leq V_{*}(t) \cdot \frac{e^{-c \mu(t)}-1}{\mu(t)}=V_{*}(t) \cdot \bar{\xi}_{-c}(t),
$$

thus property (iii) follows from (3.13) and (3.16).

In order to show that $V$ is continuous in the sense of (iv), let $t \in \mathbb{T}, x \in \mathbb{R}^{n}$ be fixed and choose $\varepsilon>0$ arbitrary. Then $\delta_{1}>0$ and $\delta_{2}>0$ must be found such that

$$
|V(\tilde{t}, \tilde{x})-V(t, x)|<\varepsilon
$$

holds for all

$$
\tilde{t}=t+v, \quad v \in A_{t}, 0 \leq v<\delta_{1}
$$

and all $\tilde{x} \in B_{\delta_{2}}(x)$, where $B_{\delta_{2}}(x)$ is the open ball centered on $x$ of radius $\delta_{2}$.

If $t \in \mathbb{T}$ is right scattered, we can always choose a suitable $\delta_{1}>0$ such that $\tilde{t}=t$ is the only point satisfying condition (3.18). Then the assertion follows since $V$ is globally Lipschitz continuous in $x$ for fixed $t \in \mathbb{T}$.

Let $t \in \mathbb{T}$ be right dense and $\tilde{t}=t+v$ for $v \in A_{t}$. Then (3.17) will follow from the inequalities

$$
\begin{aligned}
|V(\tilde{t}, \tilde{x})-V(t, x)|= & |V(t+v, \tilde{x})-V(t, x)| \leq|V(t+v, \tilde{x})-V(t+v, x)| \\
& +|V(t+v, x)-V(t+v, y(t+v, t, x))| \\
& +|V(t+v, y(t+v, t, x))-V(t, x)| .
\end{aligned}
$$

Now we can use the Lipschitz continuity of $V$ in $x$ to estimate

$$
|V(t+v, \tilde{x})-V(t+v, x)| \leq K|| x-\tilde{x} \| .
$$

Hence

$$
\left|V\left(t+v, x^{\prime}\right)-V(t+v, x)\right|<\frac{\varepsilon}{3}
$$

for all $x^{\prime} \in B_{\delta_{2}}(x)$ when $\delta_{2}<\varepsilon / 3 K$. 
In addition, the Lipschitz continuity of $V$ in $x$ also implies that

$$
|V(t+v, x)-V(t+v, y(t+v, t, x))| \leq K|| x-y(t+v, t, x)||,
$$

where, by the right continuity of $y$, we have

$$
\lim _{\nu \rightarrow 0, \nu \in A_{t}} y(t+v, t, x)=x,
$$

hence there exists a $\delta_{1}^{\prime}>0$, such that

$$
\|x-y(t+\nu, t, x)\|<\frac{\varepsilon}{3} \cdot \frac{1}{K}
$$

for all $v \in A_{t}$ with $0 \leq v<\delta_{1}^{\prime}$, which combines with (3.22) to give

$$
|V(t+\nu, x)-V(t+\nu, y(t+\nu, t, x))| \leq \frac{\varepsilon}{3}
$$

for all $v \in A_{t}$ with $0 \leq v<\delta_{1}^{\prime}$.

In order to estimate the last term in (3.19), we use the uniqueness of solutions of our initial value problem (3.1) (see [4, Theorem 8.20, Page 324]) to obtain

$$
y(t+v+\tau, t+v, y(t+v, t, x))=y(t+v+\tau, t, x) .
$$

Define

$$
\Delta V:=|V(t+v, y(t+v, t, x))-V(t, x)|
$$

that is,

$$
\Delta V=\left|\sup _{\tau \in A_{t+\nu}}\|y(t+\nu+\tau, t, x)\| e^{c \tau}-\sup _{\tau \in A_{t}}\|y(t+\tau, t, x)\| e^{c \tau}\right|
$$

Rearrangement gives

$$
\sup _{\tau \in A_{t+\nu}}\|y(t+\nu+\tau, t, x)\| e^{c \tau}=\sup _{\tau \in\{\tau \in[\nu, \infty): t+\tau \in \mathbb{T}\}}\|y(t+\tau, t, x)\| e^{c \tau} e^{-c v}
$$

so (3.28) can be rewritten as

$$
\Delta V=\left|\sup _{\tau \in\{\tau \in[v, \infty): \tau+y \in \mathbb{T}\}}\|y(t+\tau, t, x)\| e^{c \tau} e^{-c v}-\sup _{\tau \in\{\tau \in[0, \infty): t+\tau \in \mathbb{T}\}}\|y(t+\tau, t, x)\| e^{c \tau}\right| .
$$

Define $\alpha(\nu):=\sup _{\tau \in\{\tau \in[\nu, \infty): t+\tau \in \mathbb{T}\}}\|y(t+\tau, t, x)\| e^{c \tau}$ for $\nu \in A_{t}$. Then

$$
\Delta V \leq\left|\alpha(\nu) e^{-c \nu}-\alpha(0)\right| .
$$


Now $\alpha(\nu) \leq \alpha(0)$ for all $\nu \in A_{t}$, where $\nu \geq 0$ by definition. Moreover, $\alpha(\nu)$ is nonincreasing function in $\nu$ with

$$
\lim _{\nu \rightarrow 0, \nu \in A_{t}} \alpha(\nu)=\alpha(0)
$$

Hence, there exists a $\delta_{1}^{\prime \prime}>0$ such that $\Delta V<\varepsilon / 3$ for all $\nu \in A_{t}$ with $0 \leq v \leq \delta_{1}^{\prime \prime}$.

Now choose $\delta_{1}:=\min \left\{\delta_{1}^{\prime}, \delta_{1}^{\prime \prime}\right\}$. For $\tilde{t}=t+v$ with $v \in A_{t}$, where $0 \leq v<\delta_{1}$ and $\tilde{x} \in$ $B_{\delta_{2}}(x)$, combining all of the above estimates of the terms in (3.19), then gives

$$
|V(\tilde{t}, \tilde{x})-V(t, x)|<\frac{\varepsilon}{3}+\frac{\varepsilon}{3}+\frac{\varepsilon}{3}=\varepsilon
$$

which proves property (iv) and completes the proof of Theorem 3.1.

\section{Acknowledgments}

Peter Kloeden has been partly supported by the Ministerio de Educación y Ciencia Project MTM2005-01412 as well by the Programa de Movilidad del Profesorado Universitario Español y Extranjero, Grant SAB2004-0146 of the above ministerio.

\section{References}

[1] R. P. Agarwal, Difference Equations and Inequalities, Monographs and Textbooks in Pure and Applied Mathematics, vol. 155, Marcel Dekker, New York, 1992.

[2] R. P. Agarwal, M. Bohner, D. O'Regan, and A. Peterson, Dynamic equations on time scales: a survey, Journal of Computational and Applied Mathematics 141 (2002), no. 1-2, 1-26.

[3] B. Aulbach and S. Hilger, Linear dynamic processes with inhomogeneous time scale, Nonlinear Dynamics and Quantum Dynamical Systems (Gaussig, 1990), Math. Res., vol. 59, Akademie, Berlin, 1990, pp. 9-20.

[4] M. Bohner and A. Peterson, Dynamic Equations on Time Scales, Birkhäuser Boston, Massachusetts, 2001.

[5] M. Bohner and A. Peterson (eds.), Advances in Dynamic Equations on Time Scales, Birkhäuser Boston, Massachusetts, 2003.

[6] J. B. Conway, Functions of One Complex Variable I, 2nd ed., Graduate Texts in Mathematics, vol. 11, Springer, New York, 1978.

[7] A. Döffinger, Theorie dynamischer Gleichungen—ein einheitlicher Zugang zur kontinuierlichen und diskreten Dynamik, Diplomarbeit, Universität Augsburg, Augsburg, 1995.

[8] S. Hilger, Ein Maßkettenkalkül mit Anwendung auf Zentrumsmannigfaltigkeiten, Dissertation, Universität Würzburg, Würzburg, 1988.

[9] __ Analysis on measure chains — a unified approach to continuous and discrete calculus, Results in Mathematics 18 (1990), no. 1-2, 18-56.

[10] Special functions, Laplace and Fourier transform on measure chains, Dynamic Systems and Applications 8 (1999), no. 3-4, 471-488.

[11] S. Keller, Asymptotisches Verhalten invarianter Faserbündel bei Diskretisierung und Mittelwertbildung im Rahmen der Analysis auf Zeitskalen, Dissertation, Universität Augsburg, Augsburg, 1999.

[12] P. E. Kloeden and S. Hilger, The effect of time granularity on the asymptotic stability of dynamical systems, Automation and Remote Control 55 (1994), no. 9, part 1, 1293-1298 (1995). 


\section{Lyapunov functions for dynamical equations}

[13] C. Pötzsche, S. Siegmund, and F. Wirth, A spectral characterisation of exponential stability for linear time-invariant systems on time scales, Discrete and Continuous Dynamical Systems 9 (2002), 255-265.

[14] R. Remmert, Funktionentheorie, Springer, Berlin, 1995.

[15] T. Yoshizawa, Stability Theory by Liapunov's Second Method, Publications of the Mathematical Society of Japan, no. 9, The Mathematical Society of Japan, Tokyo, 1966.

[16] A. Zmorzynska, Lyapunovfunktionen auf Zeitskalen, Diplomarbeit, Johann Wolfgang Goethe Universität, Frankfurt am Main, 2004.

Peter E. Kloeden: Institut für Mathematik, Johann Wolfgang Goethe Universität, 60054 Frankfurt am Main, Germany

E-mail address: kloeden@math.uni-frankfurt.de

Alexandra Zmorzynska: Institut für Mathematik, Technische Universität Berlin, 10623 Berlin, Germany

E-mail address: zmorzynska@math.tu-berlin.de 